\title{
Quantitative characterization of hemodynamic properties and vasculature dysfunction of high-grade gliomas
}

\author{
Vijaya Nagesh, ${ }^{1}$ Thomas L. Chenevert, ${ }^{2}$ Christina I. Tsien, ${ }^{1}$ Brian D. Ross, ${ }^{2}$ Theodore S. Lawrence, ${ }^{1}$ \\ Larry Junck ${ }^{3}$ and Yue Cao, ${ }^{1,2 *}$ \\ ${ }^{1}$ Department of Radiation Oncology, University of Michigan, Ann Arbor, MI, USA \\ ${ }^{2}$ Department of Radiology, University of Michigan, Ann Arbor, MI, USA \\ ${ }^{3}$ Department of Neurology, University of Michigan, Ann Arbor, MI, USA
}

Received 21 June 2006; Revised 22 August 2006; Accepted 5 October 2006

\begin{abstract}
Aberrations in tumor and peritumoral vasculature may not be distinguishable by cerebral blood flow (CBF) or cerebral blood volume (CBV) alone. The relationships between CBF and CBV were examined to estimate vasculaturespecific hemodynamic characteristics. Twenty glioma patients were studied with dynamic susceptibility $T_{2}{ }^{*}$-weighted MRI [(dynamic contrast-enhanced magnetic resonance imaging (DSC-MRI)] before and during week 1 and 3 of radiotherapy (RT). CBF and CBV were calculated from DSC-MRI, and relationships between the two were evaluated: the physiological measure of mean transit time $(\mathrm{MTT})=\mathrm{CBV} / \mathrm{CBF}$; empirical fitting using the power law $\mathrm{CBV}=\mathrm{constant} \times(\mathrm{CBF})^{\beta}$. Three different tissue types were assessed: the Gd-enhancing tumor volume (GEV); non-enhanced abnormal tissue located beyond GEV but within the abnormal hyperintense region on FLAIR images (NEV); normal tissue in the hemisphere contralateral to the tumor (CNT). The effects of tissue types, CBV magnitudes (low, medium and high), before and during RT, on MTT and $\beta$ were analyzed by analysis of variance (ANOVA). The MTT and $\beta$ for the three tissue types were significantly different $(p<0.009)$. MTT increased from CNT $(1.60 \mathrm{~s})$ to NEV $(1.93 \mathrm{~s})$ to GEV $(2.28 \mathrm{~s})(p<0.0005)$. $\beta$ was significantly greater in GEV (1.079) and NEV (1.070) than in CNT (1.025). $\beta$ increased with increasing CBV magnitude while MTT was independent of CBV magnitude. There was a significant decrease in MTT of NEV and GEV during week 3 of RT compared with pre-RT values for all CBV magnitudes. There was a significant increase in $\beta$ during RT in the tumor and peritumor. Progressive abnormalities in vasculature and hemodynamic characteristics of the vascular bed were delineated, with significant disorder in the tumor but mild abnormality in peritumoral tissue. Copyright (C) 2007 John Wiley \& Sons, Ltd.
\end{abstract}

KEYWORDS: cerebral blood flow; cerebral blood volume; gliomas; mean transit time; radiotherapy; tumor vasculature

\section{INTRODUCTION}

One of the hallmarks of high-grade tumors is their infiltrative nature. Owing to rapid growth of the tumor, the core of the tumor becomes necrotic, while peripheral tumor cells invade into the adjacent tissue, forming a region in which tumor cells, edema and normal tissue coexist. It is therefore difficult to estimate the tumor margin for therapeutic targeting. However, given the association between tumor growth and tumor neovascularization, characterizing the functional and architectural properties of vasculature in the tumor and peritumoral region may aid in better delineation of the tumor margin.

High-grade glioma vasculature exhibits a great degree of heterogeneity in cerebral hemodynamic function and

*Correspondence to: Y. Cao, Department of Radiation Oncology, University of Michigan, 1500 E. Medical Center Drive, UH. B2C438, Box 0010, Ann Arbor, MI 48109-0010, USA.

E-mail:yuecao@umich.edu

Contract/grant sponsor: NIH; contract/grant number: 2PO1 CA59827; PO1 CA85878; R21 CA11369901. morphological abnormalities. Increased vascularity is one of the pathophysiological characteristics of high-grade gliomas. Increased vascularity is more pronounced in the contrast-enhanced rim on the post-Gd $T_{1}$-weighted image, whereas vessels in the tumor core undergo regression, resulting in a low blood volume. The peritumoral region, i.e. exterior to the enhanced rim, presumably represents the area of tumor growth and invasion into adjacent tissue. Vascular properties as well as angiogenic activity and expression of growth factors in the tumor and peritumoral region are likely to differ from each other (1-3). Thus, vascular characterization may provide valuable information on the tumor margin. In addition, tumor vasculature may play a crucial role in therapy (4). The effectiveness of radiotherapy, chemotherapy or immunotherapy is dependent on the function of the vasculature. Thus, an understanding of the structural and functional/dysfunctional characteristics of the vascular microenvironment in gliomas is essential for design of therapeutic strategies.

Physiological imaging, especially dynamic contrastenhanced (DSC) magnetic resonance (MR) imaging has 
become increasingly important in diagnosis $(5,6)$, histopathological grading (5,7-11) and management of cerebral tumors $(12,13)$. Cerebral blood volume (CBV) images estimated from DSC $T_{2}{ }^{*}$-weighted imaging have been used previously to differentiate between microvascular changes after radiotherapy and tumor recurrence (14) and to predict survival $(10,15)$. In high-grade gliomas, CBV and cerebral blood flow (CBF) are elevated in the contrast-enhanced rim and reduced in the tumor core and in the peritumoral region. Thus, whether there is alteration in the vascular properties of the peritumoral region that may be subtle and cannot be easily distinguished by $\mathrm{CBF}$ and $\mathrm{CBV}$ abnormalities alone remains to be evaluated. The objective of the present study was therefore to examine the empirical and physiological relationships between $\mathrm{CBV}$ and $\mathrm{CBF}$ to estimate vasculature-specific hemodynamic functional characteristics in order to distinguish tumor, peritumoral and normal brain tissue. Specifically, this study evaluated the mean transit time (MTT) determined as the ratio of $\mathrm{CBV}$ to $\mathrm{CBF}$, and the power exponent $\beta$, obtained from the expression, $\mathrm{CBV}=\mathrm{Constant} x(\mathrm{CBF})^{\beta}$. The concept of the power exponent was first proposed and studied by Grubb in a hypercapnic rat model (16). The study was also designed to evaluate these metrics pre radiation and to assess time- and dose-dependent changes during fractionated radiotherapy. It was hypothesized that the empirical and physiological relationships between CBV and $\mathrm{CBF}$ in the tumor and peritumoral tissue would deviate significantly from that of normal tissue, with severe dysfunction in the tumor and milder dysfunction in the peritumor vasculature; moreover, tumor vasculature will respond earlier to radiation therapy than the normal tissue vasculature.

\section{METHODS}

\section{Patients}

Twenty patients ( 13 females and seven males between the ages of 23 and 76$)$ with grade III $(n=5)$ or grade IV $(n=15)$ gliomas participated in an institutional review board approved clinical MRI study. Ten patients had subtotal tumor resection and ten had biopsy only. All patients had a minimum $4 \mathrm{cc}$ residual tumor volume, measured by volume of the contrast-enhanced region on the post-contrast $T_{1}$-weighted images. All patients underwent three-dimensional conformal radiotherapy (RT) with a median dose of $70 \mathrm{~Gy}$. MRI studies were performed prior to RT and after 1-2 weeks (after a median dose of 12 Gy) and 3-4 weeks (after a median dose of $32 \mathrm{~Gy}$ ) during RT. A summary of patient characteristics including tumor histology and treatment doses is detailed in Table 1.

\section{MRI}

The MRI protocol for all patients included axial $T_{1}$-weighted pre- and post-contrast, $T_{2}$-weighted, fluidattenuated inversion recovery (FLAIR) and dynamic contrast-enhanced (DSC) $T_{2}{ }^{*}$-weighted images. For DSC imaging, 36 volumes of dynamic $T_{2}{ }^{*}$-weighted images

Table 1. Summary of patient characteristics including tumor histology and radiation dose

\begin{tabular}{|c|c|c|c|c|c|c|}
\hline Patient number & Gender & Age (years) & Histology & Tumor grade & Surgery $(\mathrm{Sx}) /$ biopsy(Bx) & $\begin{array}{l}\text { Total accumulated } \\
\text { radiation dose }(\mathrm{Gy})\end{array}$ \\
\hline 1 & $\mathrm{~F}$ & 44 & GBM & 4 & Sx & 70 \\
\hline 2 & M & 39 & GBM & 4 & $\mathrm{Sx}$ & 72 \\
\hline 3 & $\mathrm{~F}$ & 31 & GBM & 4 & $\mathrm{Bx}$ & 72 \\
\hline 4 & M & 66 & GBM & 4 & Sx & 70 \\
\hline 5 & $\mathrm{~F}$ & 54 & GBM & 4 & $\mathrm{Bx}$ & 70 \\
\hline 6 & M & 60 & GBM & 4 & $\mathrm{Sx}$ & 75 \\
\hline 7 & $\mathrm{~F}$ & 44 & $\mathrm{AA}$ & 3 & $\mathrm{Bx}$ & 70 \\
\hline 8 & M & 61 & GBM & 4 & $\mathrm{Bx}$ & 50 \\
\hline 9 & $\mathrm{~F}$ & 68 & GBM & 4 & $\mathrm{Sx}$ & 60 \\
\hline 10 & M & 50 & GBM & 4 & $\mathrm{Bx}$ & 70 \\
\hline 11 & M & 70 & GBM & 4 & Sx & 60 \\
\hline 12 & $\mathrm{~F}$ & 63 & $\mathrm{AO}$ & 3 & $B x$ & 70 \\
\hline 13 & $\mathrm{~F}$ & 58 & GBM & 4 & $B x$ & 52 \\
\hline 14 & $\mathrm{~F}$ & 23 & GS & 4 & Sx & 66 \\
\hline 15 & $\mathrm{~F}$ & 62 & GBM & 4 & Sx & 60 \\
\hline 16 & $\mathrm{~F}$ & 48 & $\mathrm{AO}$ & 3 & $\mathrm{Bx}$ & 70 \\
\hline 17 & M & 76 & $\mathrm{AO}$ & 3 & $\mathrm{Bx}$ & 66 \\
\hline 18 & $\mathrm{~F}$ & 41 & $\mathrm{AO}$ & 3 & Sx & 70 \\
\hline 19 & $\mathrm{~F}$ & 62 & GBM & 4 & Sx & 75 \\
\hline 20 & $\mathrm{~F}$ & 73 & GBM & 4 & $B x$ & 75 \\
\hline
\end{tabular}

Gender: $\mathrm{F}=$ female, $\mathrm{M}=$ male; tumor histology: $\mathrm{AA}=$ anaplastic astrocytoma, $\mathrm{AO}=$ anaplastic oligioastrocytoma, $\mathrm{GBM}=\mathrm{glioblastoma}$ multiforme, $\mathrm{GS}=$ gliosarcoma; tumor grade: surgery $(\mathrm{Sx}=$ surgical resection, $\mathrm{Bx}=$ biopsy) and total accumulated dose received at the end of three-dimensional conformal radiation therapy treatment. 
were acquired using a gradient-echo echo-planar imaging pulse sequence with $T R=2 \mathrm{~s}, T E=60 \mathrm{~ms}$, field of view $=220 \times 220 \mathrm{~mm}^{2}$, matrix $=128 \times 128$, flip angle $=$ $60^{\circ}$ and 14 interleaved slices with $6 \mathrm{~mm}$ thickness and a $0 \mathrm{~mm}$ gap. The contrast agent Gd-DTPA $(0.1 \mathrm{~mL} / \mathrm{kg})$ was injected intravenously by a power injector at a rate of $2 \mathrm{~mL} / \mathrm{sec}$, followed immediately by $15 \mathrm{~mL}$ of saline flush at the same rate. The relative $\mathrm{CBV}$ and $\mathrm{CBF}$ in the brain and tumor were computed from DSC $T_{2}{ }^{*}$-weighted images as described below.

\section{Image processing and perfusion calculation}

All MR images acquired prior to and during RT were coregistered to the post-contrast $T_{1}$-weighted image set acquired before RT. Image registration was performed using mutual information and simplex optimization algorithms. $T_{2}{ }^{*}$-weighted images acquired with gradientecho echo-planar imaging were registered using affine transformation which included 12 parameters (nine for $3 \times 3$ rotation and shearing matrix and three for translation) to optimize and compensate for head motion, slice misalignment and geometric distortion due to magnetic field susceptibility. The CBV and CBF were computed voxel by voxel using the methods outlined by Rosen et al. (17), Østergaard et al. (18) and Liu et al. (19). Details of these calculations are given in a previous paper (15). In brief, the concentration of contrast agent in the tissue, $C_{\text {tiss }}(t)$, is the convolution of the arterial input function (AIF) and the tissue residue function $R(t)$, based upon the theory of non-diffusable tracers. The equation for $C_{\text {tiss }}(t)$ was formulated as a matrix expression and solved by a singular value decomposition (SVD) routine $(19,20)$. A small region in the middle cerebral artery was selected to calculate AIF:

$$
C_{\mathrm{tiss}}(t)=\int_{0}^{t} \operatorname{AIF}(\tau) R(t-\tau) d \tau
$$

The amplitude of the residue function is proportional to the blood flow (CBF); and the integral of $C_{\text {tiss }}(t)$ is proportional to the blood volume (CBV). The SVD was performed on a pixel-by-pixel basis to generate threedimensional CBV and CBF maps. To estimate CBV and $\mathrm{CBF}$, only the first pass peak of the bolus was used.

\section{Regions of different tissue types}

The relationships between $\mathrm{CBV}$ and $\mathrm{CBF}$ were calculated on a pixel-by-pixel basis and analyzed in three different tissue types (see below). The three tissues were: (a) the Gd-enhanced tumor volume which was defined on post-contrast $T_{1}$-weighted images and included the contrast-enhanced rim and tumor core (or surgical cavity) (GEV); (b) non-enhanced abnormal tissue that was located beyond the GEV but within the $T_{2}$ abnormal hyperintense region on FLAIR images (NEV); (c) normal tissue including all white matter and gray matter in the hemisphere contralateral to the tumor and receiving much less than $95 \%$ of the prescribed radiation dose to the tumor (CNT). For patients in whom the tumor spanned the right and left hemispheres of the brain, CNT was taken from either the posterior or anterior region of the brain that was remote from the site of the tumor. The CNT volumes ranged from 78 to $310 \mathrm{cc}$ with a mean CNT volume of $203 \mathrm{cc}$, and the mean volume of tumor and peritumor was 43 and $67 \mathrm{cc}$ respectively.

For further analysis of the effects of radiation on the relationships between CBV and CBF (see below) in all normal appearing tissue, the normal appearing tissue was divided into regions based upon received dose at the completion of RT: (a) $\leq 4 \mathrm{~Gy}$; (b) $>4 \leq 12 \mathrm{~Gy}$; (c) $>12$ $\leq 24 \mathrm{~Gy}$; (d) $>24 \mathrm{~Gy} \leq 95 \%$ of the biologically corrected dose $(\mathrm{alpha} / \mathrm{beta}=2.5)(21)$. The effect on the normal appearing tissue that received $>95 \%$ of the prescribed tumor dose was also assessed. A pictorial configuration of the various tissue types/regions studied is illustrated in Fig. 1.

\section{Normalization}

To assess the relationship between $\mathrm{CBV}$ and $\mathrm{CBF}$ over the course of RT and across patients, $\mathrm{CBV}$ and $\mathrm{CBF}$ values were normalized to white matter regions contralateral to the tumor, an approach used in previous work by the present authors (15). In brief, the white matter volumes of interest for normalization were defined in the volumes contralateral to the tumor and received $<30$ Gy biologically corrected dose at the end of RT. The reason for choosing $<30 \mathrm{~Gy}$ instead of a lower dose was to have a sufficient volume of interest for every patient. Using CBF and $\mathrm{CBV}$ values of white matter reported in the literature $(22,23)$, the mean $\mathrm{CBV}$ and $\mathrm{CBF}$ in the normal white matter volume were normalized to $100 \mathrm{~mL} / 100 \mathrm{~g}$ and $30 \mathrm{~mL} / 100 \mathrm{~g} / \mathrm{min}$ respectively. This normalization would influence the absolute value of the mean transit time (MTT $=$ the ratio of CBV to CBF, further defined below) but not affect changes in MTT from one tissue region to another, or over the course of treatment. Also, this normalization would not affect the power exponent (defined below).

\section{CBV-CBF relations}

The coupling between CBF and CBV was evaluated by two different approaches:

1. Mean transit time. The MTT was calculated on the basis of the central volume principle (24), relating $\mathrm{CBF}, \mathrm{CBV}$ and MTT as follows: $\mathrm{MTT}=\mathrm{CBV} / \mathrm{CBF}$. 


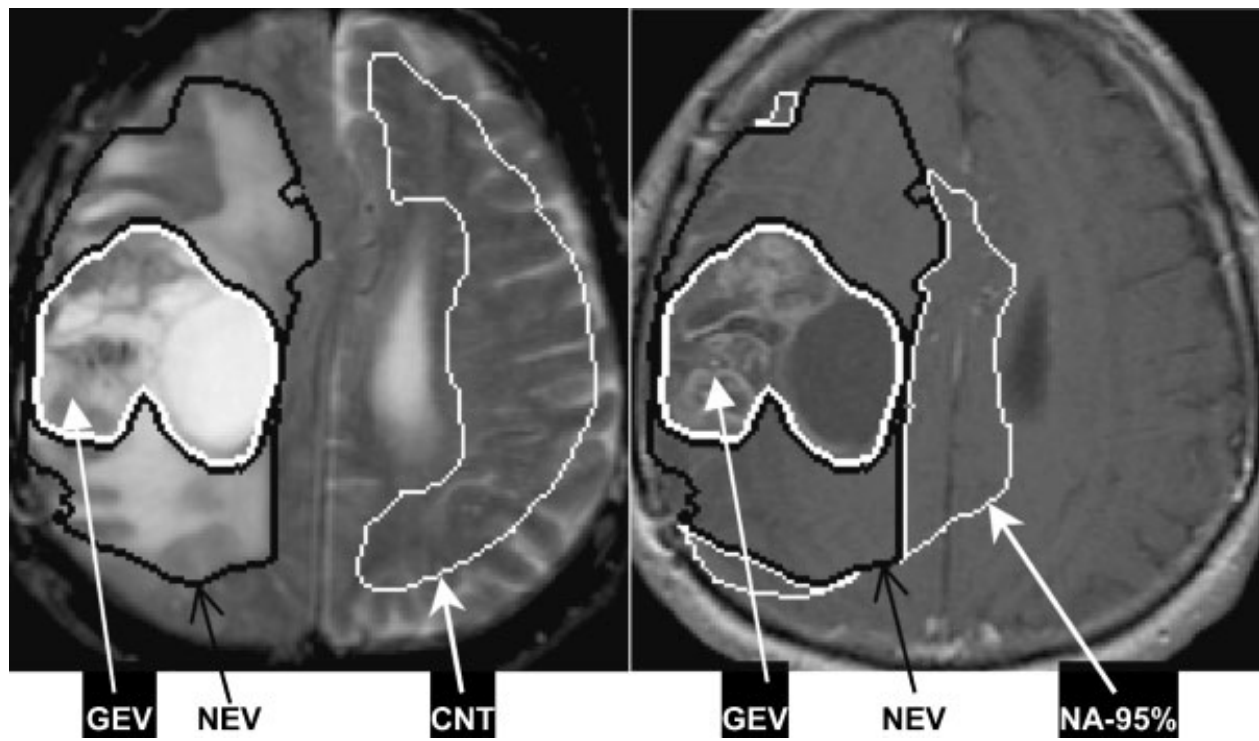

Figure 1. Illustration of the volumes of interest of the different tissue types, and the spatial relation and proximity of the volumes. FLAIR (left) and post-Gd-DTPA $T_{1}$-weighted images (right) were used to define the FLAIR tumor volume and Gd-DTPA contrast-enhanced tumor volume (GEV). The volume of non-enhanced abnormal tissue (NEV) is the difference in the FLAIR tumor volume and the GEV. The GEV is enclosed by the thick white contour, and the NEV by the black contour on both panels. CNT that is contralateral to the tumor is denoted by the white contour on the left panel. The volume of normal appearing tissue that received $95 \%$ of the biologically corrected radiation dose to the tumor (NA-95\%) is adjacent to the tumor and depicted by the outer white contour on the right panel

2. Power law. Empirical fitting of $\mathrm{CBV}$ and $\mathrm{CBF}$ using the power law $\mathrm{CBV}=A \times(\mathrm{CBF})^{\beta}$, where constants $A$ and $\beta$ are the proportionality constant and power exponent respectively. The power-law relationship was estimated by fitting the rest-state $\mathrm{CBV}$ and $\mathrm{CBF}$ data within a $5 \times 5$ voxel kernel to obtain maps of $A$ and $\beta$ using least-squares minimization. The statistical significance for fitting the $5 \times 5$ voxel was set at $p<0.05$. Therefore, voxels with $p \geq 0.05$ were discarded from the analysis. Deviation of the power exponent $\beta$ from unity indicates that the relationship between $\mathrm{CBV}$ and $\mathrm{CBF}$ has departed from the central volume principle.

\section{Definition and range of CBV magnitudes}

To assess the dependence of MTT or power exponent on CBV magnitudes, the MTT and the power exponent were further evaluated on the basis of low, medium, and high CBV magnitudes in each of the three tissue types (GEV, NEV and CNT). The CBV classification scheme used was identical to the one described in the previous study (15). Briefly, the CBV values of normal white and gray matter tissue reported in the literature were used to define the threshold values of low, medium and high CBV. The low CBV interval was characterized as CBV being $>0.2 \%$ and
$<1.75 \%$, the medium CBV range was $1.75-4 \%$ and high $\mathrm{CBV}$ was $>4 \%$. The cut-off threshold $\mathrm{CBV}$ of $0.2 \%$ was used to exclude necrotic tissue or a surgical cavity in which there was no $\mathrm{CBV}$ or very low $\mathrm{CBV}$.

\section{Statistical analysis}

Statistical analysis was performed with the commercially available Statistical Software Package for Social Sciences program (SPSS Software Products, Chicago, IL). Effects of three factors - tissue type (GEV, NEV and CNT), CBV magnitude (low, medium and high) and time of MRI scan (pre-RT, week 1 during RT and week 3 during RT) - on MTT were assessed. Three major effects on MTT and interactions between these factors were analyzed by factorial analysis of variance (ANOVA). Then, post hoc analyses were performed to evaluate differences in MTT between conditions. A $p$ value of $<0.05$ was considered significant. Similar analyses were applied to the power exponent. Descriptive statistics (mean and standard deviation) were also obtained.

In the normal appearing tissue regions, which were divided into five subregions based upon received biologically corrected doses, the effects of radiation dose and time of scan on the power exponents were also analyzed by factorial ANOVA. 


\section{RESULTS}

\section{Visualization of tumor perfusion heterogeneity}

Tumor heterogeneity in high-grade gliomas was manifested by a complex pattern of low, medium and high CBV distribution in all patients. Examples from two patients with different distribution patterns are illustrated in Fig. 2. In the first patient (Fig. 2, top row), one low-CBV region was central and presumably was predominantly necrotic tissue, while another was on the outer rim. The high-CBV region corresponded approximately to the enhancing rim. The medium-CBV regions were concentrically situated between the high-CBV region and the inner and outer low-CBV regions. In contrast, in another patient (Fig. 2, bottom row) the high-CBV region formed the outer border of the tumor, with pockets of medium and low CBV in its vicinity. Complex patterns of CBV heterogeneity were also noted in tissue adjacent to the tumor, i.e. peritumoral tissue. Perfusion heterogeneity in tumor and surrounding tissue is highly suggestive of differences in vasculature that likely contribute to distinct forms of pathology.

Some general observations can be made about spatial distributions of the low and high $\mathrm{CBV}$ in different pathological tissue. Low CBV in the tumor (GEV) was generally coincident with the tumor core which was hyperintense on the FLAIR/ $T_{2}$ images and possibly signified necrosis. High CBV in the GEV was mapped onto the contrast-enhanced rim on the post-Gd $T_{1}$-weighted image. In some patients, high CBV in NEV did not correspond to specific features on $T_{2}$ and $T_{1}$ anatomical images. Low CBV in the NEV region was coincident with the hypointense region on $T_{1}$-weighted images and the hyperintense region of the $T_{2}$-weighted images, and it was contiguous with the contrast-enhanced rim on the $T_{1}$-weighted images.

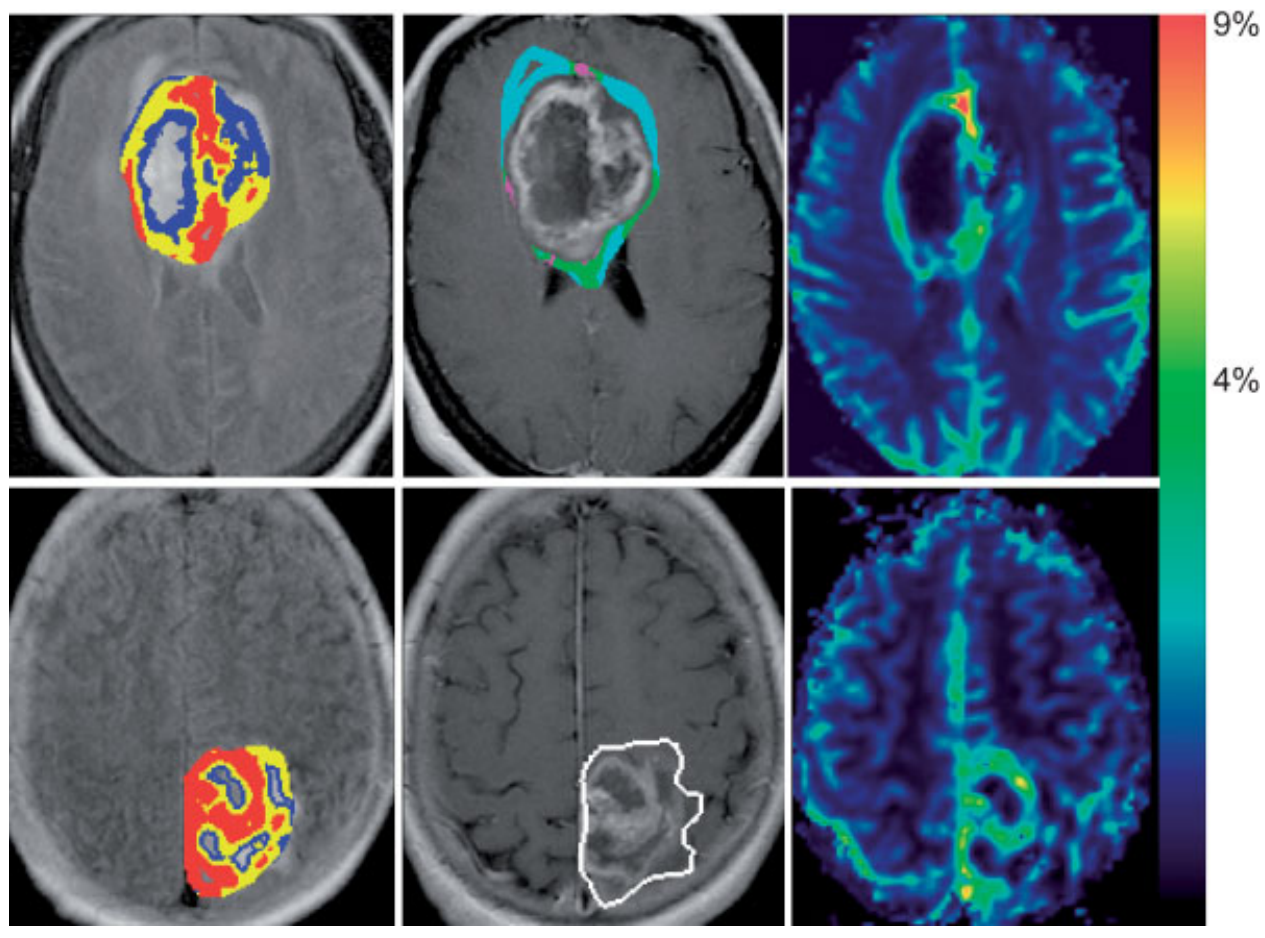

Figure 2. Examples of vascular and spatial heterogeneity in the distribution patterns of the cerebral blood volume in tumor and peritumoral tissue was observed for all patients. Images from two representative cases, patient 13 (top row) and patient 20 (bottom row) listed in Table 1 are illustrated. Shown in the left column are FLAIR images on which the low-CBV (blue), medium-CBV (yellow) and high-CBV (red) contours are overlaid. In the center column are the post-Gd $T_{1}$-weighted images. The FLAIR tumor volume comprising the tumor and peritumor tissue is represented by the white contour in the middle lower image (patient 20). An illustration of CBV heterogeneity in peritumoral tissue is shown for patient 13 (center image, top row): low CBV is represented by the cyan, medium CBV by the green and high-CBV by the magenta regions. In the right column are the color-coded CBV maps, with the color bar on the right. Note the complex patterns of tumor vasculature (top row) in which one region of low CBV was located in the tumor center while another region was on the outer rim of the tumor, the high-CBV region corresponded approximately to the enhancing rim and the medium-CBV region was located between the high-CBV and the inner and outer low-CBV regions. In the second case (bottom row), high CBV was coincident with the outer border of the tumor surrounded by compartments of low and medium CBV 
Table 2. Average of the mean transit times (MTTs) and SD for the low, medium and high CBV of the different tissue types before and during the course of radiation therapy

\begin{tabular}{|c|c|c|c|c|c|c|c|c|c|}
\hline \multirow[b]{3}{*}{ Tissue } & \multicolumn{9}{|c|}{ MTT (s) } \\
\hline & \multicolumn{3}{|c|}{ Low CBV } & \multicolumn{3}{|c|}{ Medium CBV } & \multicolumn{3}{|c|}{ High CBV } \\
\hline & Pre & Week 1 & Week 3 & Pre & Week 1 & Week 3 & Pre & Week 1 & Week 3 \\
\hline CNT & $1.62 \pm 0.18$ & $1.62 \pm 0.15$ & $1.65 \pm 0.15$ & $1.53 \pm 0.24$ & $1.53 \pm 0.21$ & $1.50 \pm 0.15$ & $1.65 \pm 0.42$ & $1.77 \pm 0.69$ & $1.56 \pm 0.24$ \\
\hline NEV & $1.89 \pm 0.33$ & $1.86 \pm 0.27$ & $1.74 \pm 0.24$ & $1.86 \pm 0.36$ & $1.86 \pm 0.24$ & $1.74 \pm 0.21$ & $2.04 \pm 0.42$ & $2.13 \pm 0.57$ & $1.86 \pm 0.27$ \\
\hline GEV & $2.19 \pm 0.60$ & $2.13 \pm 0.45$ & $1.95 \pm 0.51$ & $2.22 \pm 0.63$ & $2.16 \pm 0.48$ & $2.01 \pm 0.39$ & $2.43 \pm 0.78$ & $2.25 \pm 0.75$ & $2.10 \pm 0.54$ \\
\hline
\end{tabular}

Key to abbreviations: $\mathrm{CNT}=$ normal tissue in hemisphere contralateral to the tumor site and receiving less than $95 \%$ of the prescribed radiation dose; $\mathrm{NEV}=$ non-enhanced abnormal tissue that was located beyond the Gd-enhanced tumor volume but within the $T_{2}$ abnormal hyperintense region on FLAIR images; GEV $=$ Gd-enhanced tumor volume that was defined on post-contrast $T_{1}$-weighted images.

\section{Mean transit time}

The MTTs calculated using the central volume principle for different tissue types and CBV magnitudes before and during the course of RT are summarized in Table 2 . Factorial ANOVA indicated strong evidence of the main effects of tissue type $(p<0.0005)$ and time of the MRI scan $(p=0.002)$ but no effect of CBV magnitude $(p>0.09)$ on MTT. There was no evidence of two-way or three-way interactions between tissue type, time of scan and CBV magnitude $(p>0.2)$ (see Table 3).

Post hoc analysis revealed that MTT values were elevated significantly $(p<0.0005)$ in GEV and NEV compared with CNT, longest in GEV and shortest in CNT. Also, MTT did not change significantly after 1 week of RT but changed significantly after 3 weeks of RT. MTT at week 3 during RT was significantly decreased compared with MTT at week 1 of RT or pre-RT $(p \leq 0.02)$ (Table 3 and Fig. 3). In the GEV and the NEV, MTT of high CBV was significantly elevated compared with one with low or medium CBV $(p<0.05)$, but there was no significant difference between the MTT values of low and medium $\mathrm{CBV}(p>0.2)$ when the data were combined over all time points.

\section{Power law}

Values for the power exponent $\beta$, obtained before and during RT in the different tissue types and with the different CBV magnitudes, are summarized in Table 4. Results of the factorial ANOVA of main effects and

Table 3. Summary of factorial ANOVA of the effects of tissue type (CNT versus NEV versus GEV), CBV magnitude (low versus medium versus high) and time of MRI scan (pre versus week 1 versus week 3 during RT) on the time MTT and power exponent $\beta$, as well as interaction effects and post hoc analysis of these factors, tabulated with the level of significance indicated by $p$ values

\begin{tabular}{|c|c|c|}
\hline Test & MTT & Exponent of power fit \\
\hline \multicolumn{3}{|l|}{ Between subject tests } \\
\hline Time & $p=0.002$ & $p=0.007$ \\
\hline CBV magnitude & $p=0.098$ & $p<0.0005$ \\
\hline Tissue type & $p<0.0005$ & $p<0.0005$ \\
\hline \multicolumn{3}{|l|}{ Interaction effects - two-way } \\
\hline Time-CBV magnitude & $p=0.21$ & $p=0.745$ \\
\hline Time-tissue type & $p=0.47$ & $p=0.788$ \\
\hline CBV magnitude-tissue type & $p=0.83$ & $p<0.0005$ \\
\hline \multicolumn{3}{|l|}{ Interaction effects - three-way } \\
\hline Time-CBV magnitude-tissue type & $p=0.99$ & $p=0.99$ \\
\hline \multicolumn{3}{|l|}{ Post hoc analysis } \\
\hline \multirow[t]{3}{*}{ Time } & Pre-RT versus week 1 not significant & Pre-RT versus week $1 p=0.04$ \\
\hline & Pre-RT versus week $3 p=0.002$ & Pre-RT versus week $3 p=0.01$ \\
\hline & Week 1 versus week $3 p=0.02$ & Week 1 versus week 3 not significant \\
\hline \multirow[t]{3}{*}{ CBV magnitude } & Low versus medium not significant & Low versus medium $p<0.0005$ \\
\hline & Low versus high not significant & Low versus high $p<0.0005$ \\
\hline & Medium versus high not significant & Medium versus high $p<0.0005$ \\
\hline \multirow[t]{3}{*}{ Tissue type } & CNT versus NEV $p<0.0005$ & CNT versus NEV $p=0.002$ \\
\hline & CNT versus GEV $p<0.0005$ & CNT versus GEV $p<0.0005$ \\
\hline & NEV versus GEV $p<0.0005$ & NEV versus GEV not significant \\
\hline
\end{tabular}



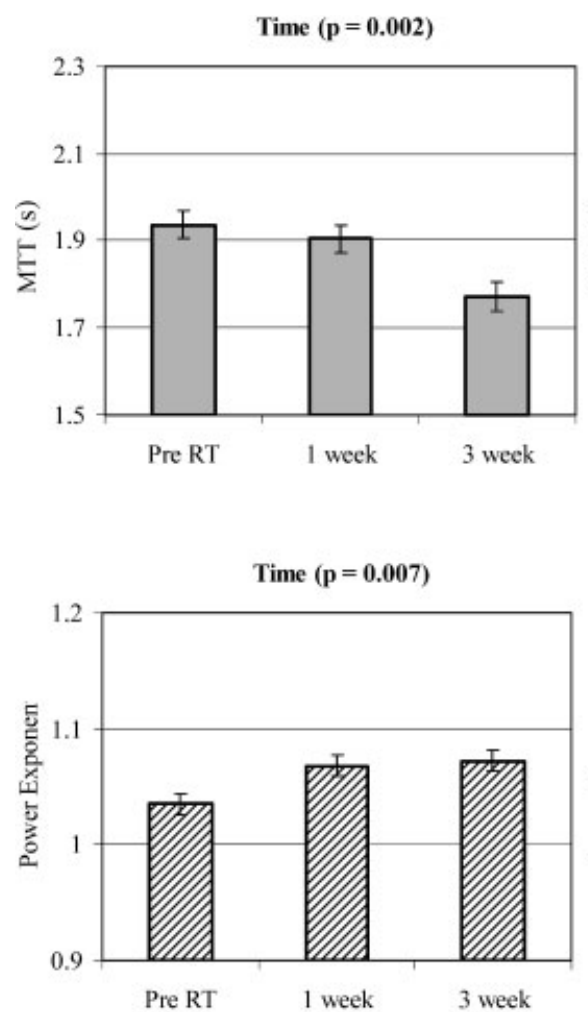

CBV Magnitude $(p=0.098)$

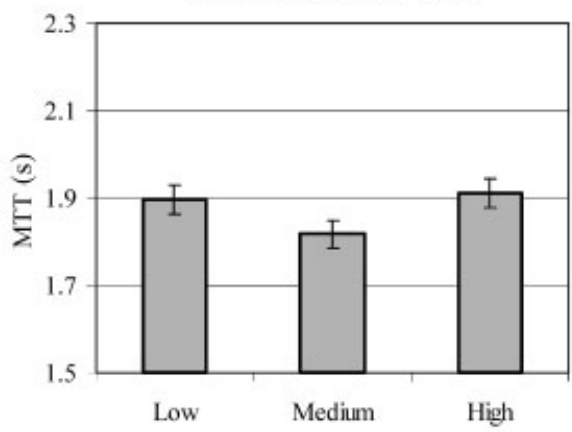

CBV Magnitude $(\mathrm{p}<0.0005)$

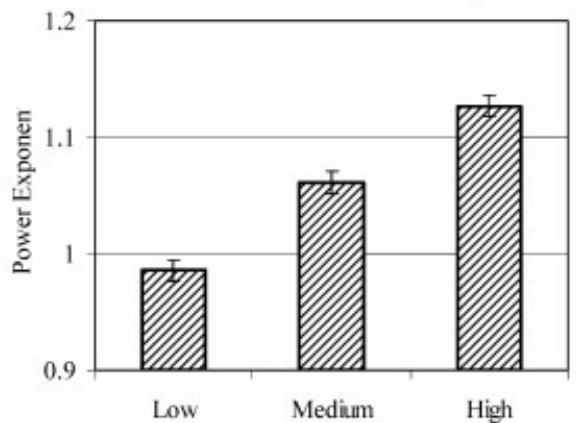

Tissue Type $(\mathrm{p}<0.0005)$

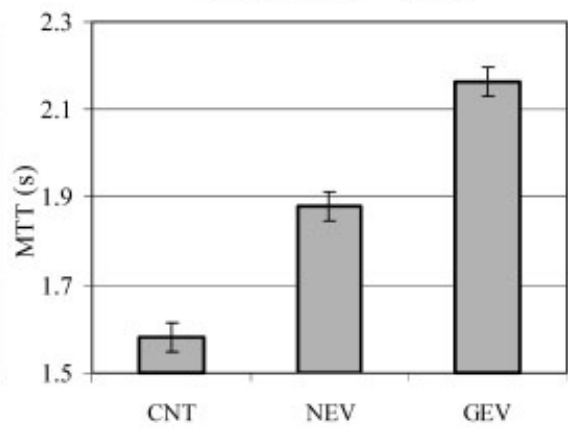

Figure 3. Factorial ANOVA was used to study the main effects of time of MRI scan in the left column (pre and weeks 1 and 3 during radiotherapy), CBV magnitude (low, medium and high) in the center column and tissue type (GEV, NEV and CNT) in the right column on the MTT (top-row graphs) and the power exponent $\beta$ (bottom-row graphs) illustrated by the bar graphs. Significance was assumed at $p<0.05$. There was strong evidence of the main effects of tissue type $(p<0.0005)$ and time of MRI scan $(p<0.002)$, but there was no effect of CBV magnitude on MTT $(p>0.09)$. There was very strong evidence of the main effects of CBV magnitude $(p<0.0005)$, tissue type $(p<0.0005)$ and time of scan $(p=0.007)$ on $\beta$ (bottom panel of graphs)

interactions between the three factors of tissue types, time of scan and CBV magnitudes on the power exponents are outlined in Table 3 . There was very strong evidence of the main effects of CBV magitudes $(p<0.0005)$, tissue types $(p<0.0005)$ and time of scan $(p=0.007)$ on the power exponent. There was a significant two-way interaction between CBV magnitudes and tissue types $(p<0.0005)$ (see Fig. 4), but interactions among the other factors were not significant.
The post hoc analysis revealed that the power exponents increased with increasing CBV $(p<0.0005)$ (Fig. 3). The power exponents in the NEV and GEV were significantly greater than that in the CNT; but the exponent in the NEV was not significantly different from that in the GEV (Fig. 3). In the GEV, the power exponent of high CBV was lower whereas the exponents of low and medium CBV were higher compared with the corresponding values in NEV and CNT, as indicated by the

Table 4. Mean \pm SD of the power exponent $\beta$ before and at weeks 1 and 3 during the course of radiotherapy for the different tissue types in the low, medium and high CBV, obtained by empirically fitting CBV and CBF. The non-linear relation between the two quantities was evaluated using the following fit: $\mathrm{CBV}=$ constant $\times(\mathrm{CBF})^{\beta}$

\begin{tabular}{|c|c|c|c|c|c|c|c|c|c|}
\hline \multirow[b]{3}{*}{ Tissue } & \multicolumn{9}{|c|}{ Power coefficient } \\
\hline & \multicolumn{3}{|c|}{ Low-CBV } & \multicolumn{3}{|c|}{ Medium-CBV } & \multicolumn{3}{|c|}{ High-CBV } \\
\hline & Pre & Week 1 & Week 3 & Pre & Week 1 & Week 3 & Pre & Week 1 & Week 3 \\
\hline CNT & $0.90 \pm 0.06$ & $0.91 \pm 0.06$ & $0.93 \pm 0.06$ & $1.02 \pm 0.08$ & $1.03 \pm 0.08$ & $1.04 \pm 0.05$ & $1.11 \pm 0.19$ & $1.13 \pm 0.11$ & $1.15 \pm 0.08$ \\
\hline $\mathrm{NEV}$ & $0.97 \pm 0.10$ & $1.00 \pm 0.12$ & $1.02 \pm 0.09$ & $1.05 \pm 0.12$ & $1.07 \pm 0.11$ & $1.08 \pm 0.09$ & $1.10 \pm 0.18$ & $1.18 \pm 0.14$ & $1.15 \pm 0.09$ \\
\hline GEV & $1.03 \pm 0.13$ & $1.07 \pm 0.13$ & $1.05 \pm 0.10$ & $1.06 \pm 0.15$ & $1.09 \pm 0.14$ & $1.11 \pm 0.14$ & $1.06 \pm 0.18$ & $1.13 \pm 0.15$ & $1.12 \pm 0.12$ \\
\hline
\end{tabular}

Key to abbreviations: $\mathrm{CNT}=$ normal tissue in hemisphere contralateral to the tumor site and receiving less than $95 \%$ of the prescribed radiation dose; $\mathrm{NEV}=$ non-enhanced abnormal tissue that was located beyond the Gd-enhanced tumor volume but within the $T_{2}$ abnormal hyperintense region on FLAIR images; GEV $=$ Gd-enhanced tumor volume that was defined on post-contrast $T_{1}$-weighted images. 


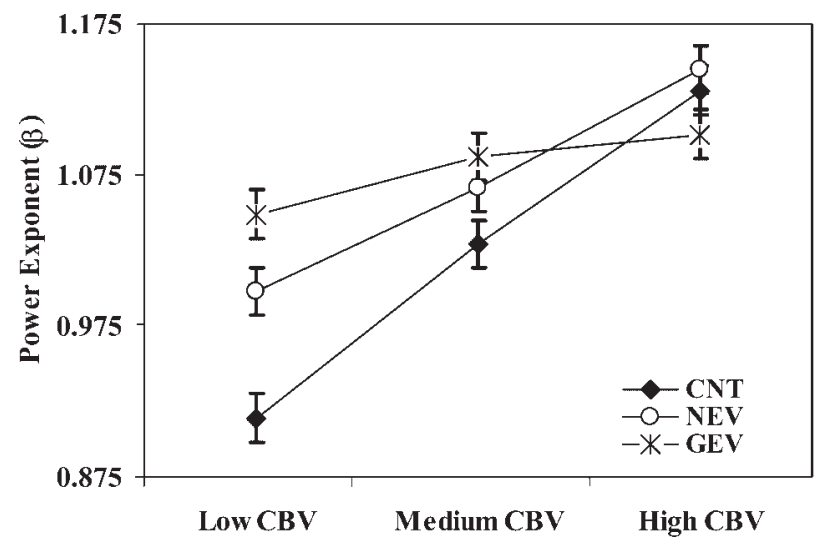

Figure 4. Factorial ANOVA of the two-way interaction between the three independent factors of CBV magnitude (low, medium and high), tissue type (GEV, NEV and CNT) and time of MRI scan (pre versus during radiation therapy) indicated that there was a significant interaction effect only between CBV magnitude (low, medium and high) and tissue type (GEV, NEV and CNT) for the power exponent dependent variable $(p<0.0005)$; all other two-way and three-way interactions for MTT and $\beta$ were not significant (Table 3). Note that, for high CBV, the power exponent in the tumor (GEV, cross symbol) was smaller than in the peritumor (NEV, open circle symbol) and normal tissue (CNT, solid diamond symbol). The power exponent of high CBV in the tumor (GEV) was smaller than that of peritumor (NEV) or normal (CNT) tissue, whereas the exponents of low and medium CBV in GEV were larger than the corresponding values in NEV and CNT

interaction of the effects between tissue type and CBV magnitude (Fig. 4). Post hoc analysis of the time of scans indicated an increase in the power exponent after RT compared with the pre-RT values $(p<0.05)$ (see Table 3).

\section{Effect of dose on power exponent in normal tissue}

The effects of radiation dose on the power exponent characteristics in normal tissue surrounding the tumor were further examined. The power exponents in normal appearing tissue were assessed in five regions that received biologically corrected doses of (a) $\leq 4 \mathrm{~Gy}$, (b) 4-12 Gy, (c) 12-24 Gy, (d) $24 \mathrm{~Gy}$ to $95 \%$ of the prescribed dose and (e) $>95 \%$ of the prescribed dose. Factorial ANOVA of the power exponent indicated no effect of dose $(p=0.42)$ or time of MRI scan $(p=0.23)$ in the normal appearing tissue.

\section{DISCUSSION}

Quantitative characterization of the physiological and empirical correlation/coupling between cerebral blood volume and cerebral blood flow provided inherently different information about tumor vasculature. The present study found that the MTT was sensitive to tissue type (normal versus abnormal) and time of MRI scan (pre-therapy versus during radiation therapy) but was independent of CBV magnitude. In contrast, the power exponent was strongly dependent on CBV magnitude (low versus medium versus high), tissue type [normal (CNT) versus both non-enhancing abnormal (NEV) and Gd-DTPA contrast-enhanced (GEV) pathologies] and time of scan (pre-therapy versus during radiation therapy or received radiation dose). There was mild but significant abnormality in the microvascular function (as measured by MTT) of peritumoral tissue (NEV) and severe dysfunction in the tumor (GEV). Prolonged MTT in tumor and peritumoral tissue when compared with normal tissue suggests increased vessel tortuosity and/or decreased blood velocity (see below). In normal tissue, the power exponent increased with increasing CBV magnitude, but in GEV the exponent was nearly constant for all CBV magnitudes, likely owing to loss of physiological distinction among arterioles, capillaries and venules in the tumor. Elevation in the low- and medium-CBV power exponent of GEV compared with that of normal tissue indicates that the tumor vascular bed provides a smaller amount of blood flow to the same amount of blood volume, most likely owing to disorganized, chaotic and irregular vessels in the tumor. Taken together, the present study suggests that the MTT and the power exponent metrics provide additional information on hemodynamic properties and vasculature dysfunction of glioma from CBV and CBF, not previously reported.

Significant differences in the MTT and power exponent of normal tissue and tumor suggest that both hemodynamic function and structural changes in the glioma vasculature affect $\mathrm{CBV}, \mathrm{CBF}$ and the relationship between CBV and CBF. The glioma vessel morphology exhibits chaotic and irregular vascular branching patterns with enlarged but variable vessel diameters and variable vessel density (25-28). The glioma vessels lack the usual distinctive anatomic features and do not conform to the physiological microvasculature of brain tissue $(27,29)$. It is therefore a challenge to assess in vivo structural and morphological changes in tumor vasculature. However, these anatomical abnormalities would be expected to be accompanied with hemodynamic abnormalities in CBF and $\mathrm{CBV}$. In the present study, the relationship between $\mathrm{CBV}$ and $\mathrm{CBF}$ was evaluated to study vascular abnormalities to measure the influence of structural abnormalities on functions of vasculature in tumor and peritumoral tissue.

A striking feature of the power exponent analysis was the ability of this metric qualitatively to differentiate between vessels on the basis of the hemodynamic functional characteristics of vessels in normal and abnormal tissue. The morphological abnormalities of the glioma vasculature, such as enlargement of the vessel 
diameter and diminishment of the physiological distinction among arterioles, capillaries and venules, can affect perfusion characteristics. Study of the power-law relationship between $\mathrm{CBV}$ and $\mathrm{CBF}$ provided insight into these abnormalities. The present data show that in normal tissue the power exponent is approximately 1 for medium CBV which roughly corresponds to gray matter, less than 1 for low CBV which mainly represents white matter (Table 4) and greater than 1 for high CBV which mainly represents macrovessels. Large vessels are typically located at the surface of the brain, and gray matter is primarily in the brain cortex, but, owing to the proximity of gray matter to large vessels at the surface of the brain, there could be some contamination from the large vessels with a partial volume effect, resulting in a power exponent higher than the expected value of 1 for gray matter. These data indicate that the relationship between $\mathrm{CBF}$ and $\mathrm{CBV}$ differs in normal brain, depending on the presence of microvasculature, macrovasculature or a mix of the two within a voxel. In contrast, the power exponents in GEV were greater than 1 for all CBV magnitudes (Fig. 4), suggesting that the behavior of the tumor vasculature differs from that of microvascular structures, especially for low and medium CBV. However, combining the observations of the power exponent and the MTT, the vasculature in the tumor is substantially different from macrovasculature in normal brain, as suggested by the elevated MTT in the tumor for all CBV magnitudes compared with both micro- and macrovasculature in normal brain. In addition, in the NEV region, the power exponents of low and medium $\mathrm{CBV}$ were intermediate between those of CNT and GEV (Fig. 4), suggesting that the microvasculature in the peritumoral region undergoes abnormal changes, possibly modified by infiltrated tumor cells. In sum, the power exponent is a metric that provides qualitative assessment of structural and anatomical changes in the vessel.

Irregular and disorganized glioma vessel architecture can greatly affect the hemodynamic functional efficiency of the tumor vasculature, which can be assessed by the MTT (the ratio of $\mathrm{CBV}$ to $\mathrm{CBF}$ ). It was found that the MTTs in tumor (GEV) and peritumor (NEV) were prolonged relative to the MTT in normal tissue (CNT), signifying diffuse hemodynamic impairments from the solid tumor to peritumoral tissue (Table 2 and Fig. 3). The prolonged MTT in the tumor and the peritumoral tissue is possibly due to the disordered and irregular branching of the tumor vessels, resulting in increased tortuosity of the vasculature. The increased MTT in abnormal tissue could represent two conditions: (1) regions with low CBV and very low $\mathrm{CBF}$; (2) regions with high $\mathrm{CBV}$ and little change in $\mathrm{CBF}$. As pointed out earlier, there are two regions in which $\mathrm{CBV}$ is low and MTT is elevated. One is in the GEV and coincident with the tumor core (but excluding necrosis and surgical cavity) (see the methods section). In the tumor core, the vasculature has been described as regressed with decreased vessel density (26).
The other is in the NEV region in which there coexist tumor cells, edema and normal tissue. There are no previous reports that describe any vascular dysfunction and morphological abnormality in the vasculature of the peritumoral region (NEV) except for a decrease in CBV which can be caused by an increase in intracranial pressure owing to edema formation but without vascular dysfunction and morphological changes in vasculature (26). A decrease in both CBF and CBV in NEV could also be related to expansion of tissue by edema or by infiltrating tumor cells in the absence of new vessels. The present findings of the prolonged MTT and the elevated power exponent in the NEV region compared with normal tissue indicate that the vasculature is both functionally and structurally abnormal. This region is likely a dynamic growth region that will expand into surrounding tissue. In the tumor, the region that has both an elevated MTT and CBV was coincident with the contrast-enhanced rim on the post-Gd $T_{1}$-weighted images, indicating maximum vascularity and perfusion deficiency. These facts may signify the growth of newly formed vessels that lack the normal vessel structure, suggesting angiogenesis. The rapid growth of new vessels and loss of existing blood vessels will likely contribute to fluctuations in blood flow patterns of the tumor. These observations point to MTT as a functional metric of tissue vasculature.

Radiation injury to vasculature generally is long latent after completion of radiation therapy. A low irradiation dose that is insufficient to produce white matter necrosis may cause vascular abnormality owing to the high radiosensitivities of vascular cells (30). Endothelial cells are most sensitive to irradiation of all the cells that constitute the vessel wall. Morphological analysis of microvessels of irradiated rat cervical spinal cord showed a reduction in endothelial cells only in white matter 3 months after radiation (31). An increase in vascular permeability has been observed in animal models (32) and in patients (33) during and shortly after radiation therapy, which may be a transient effect. The increase in vascular permeability leads to edema and fibrin deposition in the interstitial space, and blood vessel walls and extravagated fibrin are subsequently replaced by collagen fibers, followed by atrophy of parenchymal tissue (30). However, how radiation produces disruption of $\mathrm{BBB}$ is unresolved, and the precise mechanism remains to be determined.

The MTT and power exponent in GEV and NEV were both affected during the early phase of radiation, signifying that vascular functional changes are accompanied with morphological changes. In GEV and NEV, the MTT was reduced significantly at week 3 of RT but not at week 1 of RT, suggesting improvement in function of the vasculature at week 3 of RT; meanwhile, the power exponents increased significantly at week 1 and changed little at week 3 of RT. In contrast, there were no changes in either the power exponent or the MTT in contralateral 
normal tissue. Taken together, these data suggest that there were no important effects of radiation on the cerebral hemodynamic properties of normal vasculature during the first 3 weeks of radiation. However, there were significant changes in hemodynamic function of the tumor and peritumor vasculature during the first 3 weeks of RT.

Cerebral blood volume can be under- or overestimated in regions of the tumor where there is breakdown of the blood-brain/tumor barrier. In these regions, extravasation of the contrast agent into the interstitium can result in $T_{1}$ shortening, thereby causing underestimation of CBV. In addition, extravascular contrast concentrations can be miscounted as intravascular contrasts, resulting in an overestimation. However, both the $T_{1}$ effect and the extravascular contribution can be minimized. Firstly, a gradient-echo pulse sequence with a longer $T R$ of $2 \mathrm{~s}$ and a smaller flip angle of $60^{\circ}$ were chosen in order to minimize $T_{1}$ effects. When visually inspecting DSC time courses in the tumor, overshooting in the tails of the time courses was never seen, suggesting that there were minimum $T_{1}$ effects. Secondly, only the first pass was used to estimate $\mathrm{CBV}$ and $\mathrm{CBF}$, which minimized potential overestimation and underestimation in respective $\mathrm{CBV}$ and $\mathrm{CBF}$, compared with using the entire DSC time courses.

The absolute values of the MTT and power exponent can be influenced by several factors involving imaging technologies and image processing. Firstly, the CBV and CBF estimated by dynamic contrast-enhanced $T_{2}{ }^{*}$-weighted imaging are relative measures of these parameters. In order to obtain MTT, both $\mathrm{CBV}$ and $\mathrm{CBF}$ were normalized to normal white matter receiving less than $30 \mathrm{~Gy}$. Scaling factors were chosen for CBV and CBF on the basis of values reported in the literature for white matter $(22,23,34)$. The range of CBV and CBF values for white matter reported in the literature is considerable, depending upon the imaging technology used, the image resolution and the subject/patient conditions (20,35-40). As in their previous study, the present authors chose a normal white matter CBV of $1 \%$ and a CBF of $30 \mathrm{~mL} /$ $100 \mathrm{~g} / \mathrm{min}$ (15). The mean MTT in normal tissue from the cohort of 20 patients in this study was $1.60 \pm 0.28 \mathrm{~s}$. Nevertheless, assessment of the MTT changes over different tissue types, in response to different radiation doses, and dependency upon CBV magnitudes should not be affected by the global scaling factor. Furthermore, the power exponent is absolutely independent of the scaling factor. Secondly, it is generally assumed that a gradient-echo pulse sequence is not selective for vessel size, thus hindering discrimination between microvasculature and macrovasculature in normal tissue, and hence CBV estimated by a gradient-echo pulse sequence is more representative of total CBV $(41,42)$. Given that the physiological distinction between arterioles, capillaries and venules is diminished in glioma vasculature, and that the diameter of tumor vessels can increase up to $40 \mu \mathrm{m}$ $(43,44)$, it appears to be favorable to use the gradient-echo pulse sequence in high-grade gliomas, whereas a spin-echo pulse sequence that is selectively sensitive to vessels with a diameter less than that of capillaries (43) may lose sensitivity to image the enlarged vessels in the tumor (42). Thirdly, the power-law relationship between $\mathrm{CBV}$ and $\mathrm{CBF}$ has been investigated previously $(15,16,24,45,46)$. In 1954, Meier and Zierler (24) proposed the central volume principle as the linear relation between $\mathrm{CBV}$ and $\mathrm{CBF}$ in which the power exponent is unity. This linear relation has been demonstrated to be valid in a large range of CBVs and CBFs and in the rest state by experimental observations $(15,24,47,48)$. However, under different physiological or pathological conditions, the relationship between CBV and $\mathrm{CBF}$ might change. In 1974, Grubb et al. (16) investigated the relationship between the relative changes in $\mathrm{CBV}$ and $\mathrm{CBF}$ under hypercapnic challenges, and found the power exponent to be 0.38 . This non-linearity between $\mathrm{CBV}$ and $\mathrm{CBF}$ is explained as the $\mathrm{CO}_{2}$-induced increase in $\mathrm{CBF}$ owing to decrease in cerebral vascular resistance occurring as a result of vasodilation (16). Grubb's relationship is also found to be true under neuronal activation (49). The previous study by the present authors (15) found that the rest-state regional $\mathrm{CBV}$ and $\mathrm{CBF}$ were linearly correlated in high-grade gliomas, and the linear correlation was approximately maintained even after radiation. In the present study, whether there was a deviation from the central volume principle in high-grade gliomas and after radiation was evaluated. Thus, $\beta$ was evaluated for rest-state CBV and $\mathrm{CBF}$ in spatially adjacent voxels. Finally, the authors would like to point out that their methodology can differentiate the power exponents of gray and white matter (the power exponent was larger in medium-CBV regions, which are predominantly gray matter, than in low-CBV regions, which are predominantly white matter) (see Table 4) and delineate the subtle differences in the MTT between normal gray matter and white matter (MTT was slightly longer in low-CBV regions, primarily white matter, than in medium-CBV regions, predominantly gray matter) (see Table 2).

\section{CONCLUSIONS}

The cerebral hemodynamic functional and structural/ morphological characteristics of tumor vasculature in high-grade gliomas can be assessed quantitatively and/or qualitatively by the two metrics that are robustly obtained from MRI-estimated cerebral blood volume and cerebral blood flow. With these two metrics - the mean transit time and the power exponent, progressive abnormalities in vasculature function characteristics as a result of alterations in structural properties of the tumor vascular bed were detected, with more striking abnormalities in tumor and milder abnormalities in peritumoral tissue. The vasculature of normal appearing tissue surrounding the 
tumor was not affected by radiation dose delivered to the tissue in the first 3 weeks during the course of radiation therapy. Non-invasive surrogate markers of the vasculature that provide information about the structural properties as well as hemodynamic function are of value in targeting and evaluating therapy.

\section{Acknowledgements}

The authors thank Zhou Shen for computer software support and Dan Tatro and Jonathan Alspaugh for dosimetry assistance.

\section{REFERENCES}

1. Bello L, Francolini M, Marthyn P, Zhang J, Carroll RS, Nikas DC, Strasser JF, Villani R, Cheresh DA, Black PM. Alpha(v)beta3 and alpha(v)beta5 integrin expression in glioma periphery. Neurosurgery 2001; 49: 380-389, discussion 390.

2. Dafni H, Israely T, Bhujwalla ZM, Benjamin LE, Neeman M. Overexpression of vascular endothelial growth factor 165 drives peritumor interstitial convection and induces lymphatic drain: magnetic resonance imaging, confocal microscopy, and histological tracking of triple-labeled albumin. Cancer Res. 2002; 62: 6731-6739.

3. Vaithilingam IS, McDonald W, Stroude EC, Cook RA, Del Maestro RF. Proteolytic activity during the growth of C6 astrocytoma in the murine spheroid implantation model. Can. J. Neurol. Sci. 1992; 19: 17-22.

4. Folkman J. Tumor angiogenesis: therapeutic implications. N. Engl. J. Med. 1971; 285: 1182-1186.

5. Aronen HJ, Gazit IE, Louis DN, Buchbinder BR, Pardo FS, Weisskoff RM, Harsh GR, Cosgrove GR, Halpern EF, Hochberg $\mathrm{FH}$, et al. Cerebral blood volume maps of gliomas: comparison with tumor grade and histologic findings. Radiology 1994; 191: $41-51$.

6. Sugahara T, Korogi Y, Shigematsu Y, Liang L, Yoshizumi K, Kitajima M, Takahashi M. Value of dynamic susceptibility contrast magnetic resonance imaging in the evaluation of intracranial tumors. Top. Magn. Reson. Imaging 1999; 10: 114-124.

7. Sugahara T, Korogi Y, Kochi M, Ikushima I, Hirai T, Okuda T, Shigematsu Y, Liang L, Ge Y, Ushio Y, Takahashi M. Correlation of MR imaging-determined cerebral blood volume maps with histologic and angiographic determination of vascularity of gliomas. AJR Am. J. Roentgenol. 1998; 171: 1479-1486.

8. Knopp EA, Cha S, Johnson G, Mazumdar A, Golfinos JG, Zagzag D, Miller DC, Kelly PJ, Kricheff II. Glial neoplasms: dynamic contrast-enhanced $T_{2}{ }^{*}$-weighted MR imaging. Radiology 1999; 211: 791-798.

9. Donahue KM, Krouwer HG, Rand SD, Pathak AP, Marszalkowski CS, Censky SC, Prost RW. Utility of simultaneously acquired gradient-echo and spin-echo cerebral blood volume and morphology maps in brain tumor patients. Magn. Reson. Med. 2000; 43: 845-853.

10. Lev MH, Ozsunar Y, Henson JW, Rasheed AA, Barest GD, Harsh GRt, Fitzek MM, Chiocca EA, Rabinov JD, Csavoy AN, Rosen BR, Hochberg FH, Schaefer PW, Gonzalez RG. Glial tumor grading and outcome prediction using dynamic spin-echo MR susceptibility mapping compared with conventional contrast-enhanced MR: confounding effect of elevated rCBV of oligodendrogliomas [corrected]. AJNR Am. J. Neuroradiol. 2004; 25: 214-221.

11. Mills SJ, Patankar TA, Haroon HA, Baleriaux D, Swindell R, Jackson A. Do cerebral blood volume and contrast transfer coefficient predict prognosis in human glioma? AJNR Am. J. Neuroradiol. 2006; 27: 853-858.
12. Albert FK, Forsting M, Sartor K, Adams HP, Kunze S. Early postoperative magnetic resonance imaging after resection of malignant glioma: objective evaluation of residual tumor and its influence on regrowth and prognosis. Neurosurgery 1994; 34: 45-60, discussion 60-41.

13. Schmainda KM, Rand SD, Joseph AM, Lund R, Ward BD, Pathak AP, Ulmer JL, Badruddoja MA, Krouwer HG. Characterization of a first-pass gradient-echo spin-echo method to predict brain tumor grade and angiogenesis. AJNR Am. J. Neuroradiol. 2004; 25: $1524-1532$.

14. Aronen HJ, Glass J, Pardo FS, Belliveau JW, Gruber ML, Buchbinder BR, Gazit IE, Linggood RM, Fischman AJ, Rosen BR, et al. Echo-planar MR cerebral blood volume mapping of gliomas. Clinical utility. Acta Radiol. 1995; 36: 520-528.

15. Cao Y, Tsien CI, Nagesh V, Junck L, Ten Haken R, Ross BD, Chenevert TL, Lawrence TS. Clinical investigation survival prediction in high-grade gliomas by MRI perfusion before and during early stage of RT. Int. J. Radiat. Oncol. Biol. Phys. 2006; 64: $876-885$.

16. Grubb RL Jr, Raichle ME, Eichling JO, Ter-Pogossian MM. The effects of changes in $\mathrm{PaCO} 2$ on cerebral blood volume, blood flow, and vascular mean transit time. Stroke 1974; 5: 630-639.

17. Rosen BR, Belliveau JW, Buchbinder BR, McKinstry RC, Porkka LM, Kennedy DN, Neuder MS, Fisel CR, Aronen HJ, Kwong KK, et al. Contrast agents and cerebral hemodynamics. Magn. Reson. Med. 1991; 19: 285-292.

18. Ostergaard L, Weisskoff RM, Chesler DA, Gyldensted C, Rosen BR. High resolution measurement of cerebral blood flow using intravascular tracer bolus passages. Part I: mathematical approach and statistical analysis. Magn. Reson. Med. 1996; 36: 715-725.

19. Liu HL, Pu Y, Liu Y, Nickerson L, Andrews T, Fox PT, Gao JH. Cerebral blood flow measurement by dynamic contrast MRI using singular value decomposition with an adaptive threshold. Magn. Reson. Med. 1999; 42: 167-172.

20. Ostergaard L, Sorensen AG, Kwong KK, Weisskoff RM, Gyldensted C, Rosen BR. High resolution measurement of cerebral blood flow using intravascular tracer bolus passages. Part II: experimental comparison and preliminary results. Magn. Reson. Med. 1996; 36: 726-736.

21. Steel GG. Basic Clinical Radiobiology. Arnold Publishers: London, UK, 2002.

22. Derdeyn CP, Videen TO, Yundt KD, Fritsch SM, Carpenter DA, Grubb RL, Powers WJ. Variability of cerebral blood volume and oxygen extraction: stages of cerebral haemodynamic impairment revisited. Brain 2002; 125: 595-607.

23. Meyer JS, Hayman LA, Amano T, Nakajima S, Shaw T, Lauzon P, Derman S, Karacan I, Harati Y. Mapping local blood flow of human brain by CT scanning during stable xenon inhalation. Stroke 1981; 12: 426-436.

24. Meier P, Zierler KL. On the theory of the indicator-dilution method for measurement of blood flow and volume. J. Appl. Physiol. 1954; 6: 731-744.

25. Zama A, Tamura M, Inoue HK. Three-dimensional observations on microvascular growth in rat glioma using a vascular casting method. J. Cancer Res. Clin. Oncol. 1991; 117: 396-402.

26. Vajkoczy P, Schilling L, Ullrich A, Schmiedek P, Menger MD. Characterization of angiogenesis and microcirculation of highgrade glioma: an intravital multifluorescence microscopic approach in the athymic nude mouse. J. Cereb. Blood Flow Metab. 1998; 18: 510-520.

27. Vajkoczy P, Menger MD. Vascular microenvironment in gliomas. J. Neurooncol. 2000; 50: 99-108.

28. Dewhirst MW, Tso CY, Oliver R, Gustafson CS, Secomb TW, Gross JF. Morphologic and hemodynamic comparison of tumor and healing normal tissue microvasculature. Int. J. Radiat. Oncol. Biol. Phys. 1989; 17: 91-99.

29. Jain RK. Determinants of tumor blood flow: a review. Cancer Res. 1988; 48: 2641-2658.

30. Okada S, Okeda R. Pathology of radiation myelopathy. Neuropathology 2001; 21: 247-265.

31. Stewart PA, Vinters HV, Wong CS. Blood-spinal cord barrier function and morphometry after single doses of X-rays in rat spinal cord. Int. J. Radiat. Oncol. Biol. Phys. 1995; 32: 703-711.

32. Yuan H, Gaber MW, McColgan T, Naimark MD, Kiani MF, Merchant TE. Radiation-induced permeability and leukocyte 
adhesion in the rat blood-brain barrier: modulation with antiICAM-1 antibodies. Brain Res. 2003; 969: 59-69.

33. Cao Y, Tsien CI, Shen Z, Tatro DS, Ten Haken R, Kessler ML, Chenevert TL, Lawrence TS. Use of magnetic resonance imaging to assess blood-brain/blood-glioma barrier opening during conformal radiotherapy. J. Clin. Oncol. 2005; 23: 4127-4136.

34. Ito H, Kanno I, Kato C, Sasaki T, Ishii K, Ouchi Y, Iida A, Okazawa H, Hayashida K, Tsuyuguchi N, Ishii K, Kuwabara Y, Senda M. Database of normal human cerebral blood flow, cerebral blood volume, cerebral oxygen extraction fraction and cerebral metabolic rate of oxygen measured by positron emission tomography with 150-labelled carbon dioxide or water, carbon monoxide and oxygen: a multicentre study in Japan. Eur. J. Nucl. Med. Mol. Imaging 2004; 31: 635-643.

35. Leenders KL, Perani D, Lammertsma AA, Heather JD, Buckingham P, Healy MJ, Gibbs JM, Wise RJ, Hatazawa J, Herold S, et al. Cerebral blood flow, blood volume and oxygen utilization. Normal values and effect of age. Brain 1990; 113(Pt 1): 27-47.

36. Koshimoto Y, Yamada H, Kimura H, Maeda M, Tsuchida C, Kawamura Y, Ishii Y. Quantitative analysis of cerebral microvascular hemodynamics with $T_{2}$-weighted dynamic MR imaging. J. Magn. Reson. Imaging 1999; 9: 462-467.

37. Isaka Y, Itoi Y, Imaizumi M, Ashida K, Okamoto M, Iiji O. Quantitation of rCBF by 99mTc-hexamethylpropyleneamine oxime single photon emission computed tomography combined with 133Xe CBF. J. Cereb. Blood Flow Metab. 1994; 14: 353-357.

38. Petrella JR, DeCarli C, Dagli M, Grandin CB, Duyn JH, Frank JA, Hoffman EA, Theodore WH. Age-related vasodilatory response to acetazolamide challenge in healthy adults: a dynamic contrast-enhanced MR study. AJNR Am. J. Neuroradiol. 1998; 19: 39-44.

39. Schreiber WG, Guckel F, Stritzke P, Schmiedek P, Schwartz A, Brix G. Cerebral blood flow and cerebrovascular reserve capacity: estimation by dynamic magnetic resonance imaging. J. Cereb. Blood Flow Metab. 1998; 18: 1143-1156.
40. Speck O, Chang L, DeSilva NM, Ernst T. Perfusion MRI of the human brain with dynamic susceptibility contrast: gradient-echo versus spin-echo techniques. J. Magn. Reson. Imaging 2000; 12: 381-387.

41. Weisskoff RM, Zuo CS, Boxerman JL, Rosen BR. Microscopic susceptibility variation and transverse relaxation: theory and experiment. Magn. Reson. Med. 1994; 31: 601-610.

42. Boxerman JL, Hamberg LM, Rosen BR, Weisskoff RM. MR contrast due to intravascular magnetic susceptibility perturbations. Magn. Reson. Med. 1995; 34: 555-566.

43. Deane BR, Lantos PL. The vasculature of experimental brain tumours. Part 1. A sequential light and electron microscope study of angiogenesis. J. Neurol. Sci. 1981; 49: 55-66.

44. Deane BR, Lantos PL. The vasculature of experimental brain tumours. Part 2. A quantitative assessment of morphological abnormalities. J. Neurol. Sci. 1981; 49: 67-77.

45. Brown GG, Eyler Zorrilla LT, Georgy B, Kindermann SS, Wong $\mathrm{EC}$, Buxton RB. Bold and perfusion response to finger-thumb apposition after acetazolamide administration: differential relationship to global perfusion. J. Cereb. Blood Flow Metab. 2003; 23: 829-837.

46. Hoge RD, Atkinson J, Gill B, Crelier GR, Marrett S, Pike GB. Linear coupling between cerebral blood flow and oxygen consumption in activated human cortex. Proc. Natl Acad. Sci. USA 1999; 96: 9403-9408.

47. Smith AL, Neufeld GR, Ominsky AJ, Wollman H. Effect of arterial $\mathrm{CO}_{2}$ tension on cerebral blood flow, mean transit time, and vascular volume. J. Appl. Physiol. 1971; 31: 701-707.

48. Risberg J, Ingvar DH. Regional changes in cerebral blood volume during mental activity. Exp. Brain Res. 1968; 5: 72-78.

49. Ito H, Takahashi K, Hatazawa J, Kim SG, Kanno I. Changes in human regional cerebral blood flow and cerebral blood volume during visual stimulation measured by positron emission tomography. J. Cereb. Blood Flow Metab. 2001; 21: 608-612. 